\title{
Design of Collection Terminal for CAN Bus Agricultural Temperature Monitoring System Based on VB
}

\author{
Huihui Tian, Xingda Zhang, Qian Liu \\ Engineering institute, University of Jinan Quancheng College, Penglai, 265600
}

Keywords: digital agriculture; upper computer; VB6.0; collection terminal

\begin{abstract}
Aiming at the existing agricultural management, the use of information technology means, and the lack of real-time collection, transmission, management and feedback, based on the design of a VB (Visual Basic) 6 CAN (Controller Area Network, controller area network) bus temperature monitoring system of agricultural data collection terminal, this paper introduces the host computer for the development and use of intelligent CAN node temperature collection terminal design. The terminal is the programming design with the use of VB6.0 software Windows environment, multi node data acquisition, storage, processing, and decision-making interface, mainly in the development environment of temperature acquisition, temperature real-time monitoring, uploaded to the CAN node, and the CAN bus communication parameter configuration. The system has good portability and versatility, and runs well in the agricultural greenhouse environment.
\end{abstract}

\section{Introduction}

In 1998, American vice president Gore proposed the "Digital Earth". Then, some countries have carried out the research on digital ecology, which make the construction of ecological environment develop gradually to the direction of network and digitalization. Germany, Brazil, Japan, Korea and Mexico have their own thematic or comprehensive long-term ecosystem research and observation network systems in the United States, Britain and Southeast Asia. At the ninth meeting of the Chinese Academy of Sciences and the fourth academician's Congress of the Chinese Academy of engineering, China has put forward the strategy of "Digital China". The "digital society", "digital agriculture", "digital forestry", "digital water", "digital culture" and "digital logistics" to explore and practice in our country like a raging fire of [1].

The so-called digital agriculture. It is to digitize agriculture and make use of high and new technology, such as computer, communication and network, automation, and agronomy.

The subject combined to achieve in the agricultural production process on real time monitoring from macro to micro level, in order to achieve the level of the corresponding environment for regular access to information, in order to carry out the corresponding decision, to achieve good results of [2].

Digitalization is an important index to measure the development degree of an industry. Agriculture is the lowest digital level in all major industries. However, China's agriculture is also developing from the traditional agricultural model to the digital and precise modern agriculture. Precision agriculture will be the future development direction of China's agriculture.

The use of sensors, video surveillance and monitoring of crop planting environment, construction of data transmission and storage system, improve the environment for the growth of seeding, transplanting, grafting, germination and seedling production of crops, intelligent management. It's part of digital agriculture.

The construction of the Agricultural Internet of things is connected to the information network to carry out digital monitoring and management. With the networking technology, combined with modern communication technology, information management technology to change the traditional agricultural management, increasing recognition, monitoring, evaluation objective and management ability, reduce the human factors are prone to error estimation, error, mistake operation, improve the management level of standardization, science, is an important topic of research and development 
the direction of the digital management of agriculture. To explore the best economic and social benefits of the Agricultural Internet of things technology system is the technical problem that we need to solve at present [3].

In many environmental factors in agriculture, agricultural temperature is one of the key factors, the existing agricultural management and use of information technology means the lack of real-time collection, transmission, management and feedback, lack of information technology and automatic control technology based on the use of electronic equipment, information technology, modern communication technology and automation technology, design a set of efficient, stable, practical, low cost CAN bus temperature monitoring system of agriculture,

CAN is currently the only serial field bus with international standard, multi master mode, the communication distance is long and flexible, high communication rate, node capacity, packet transmission information by low frequency interference, to ensure that the data transmission time is short, low error rate, error detection and good effect, which makes the CAN Technology in the world the highly sought after, this technology was applied in many fields, CAN bus temperature monitoring system of agricultural official of the technology is very good use of [4].

This paper mainly introduces the design, development and application of the data acquisition terminal of the host computer. The CAN temperature monitoring system has been introduced in my earlier article, but is not being mentioned here.

\section{A Brief Introduction of Terminal System}

VB6.0 is the most widely used language in the world. It is an event driven programming language, which is developed by Microsoft Corp, which includes development environment. It comes from BASIC programming language. VB has graphical user interface (GUI) and rapid application development (RAD) system. It can easily connect DAO with RDO or ADO, or create ActiveX controls easily. Programmers can easily use the components provided by VB to quickly build an application. So it is easy to manage and maintain [5] by using VB.

The system is mainly composed of management landing interface, general system platform, temperature data acquisition, storage, search, decision processing, CAN data acquisition, communication parameter configuration and other interfaces.

The user login interface includes administrator login, including common contact log settings; temperature acquisition interface including the necessary temperature acquisition and real-time display and analysis of data, and print output function; interface includes temperature inquiry, analysis and processing, print output and other functions, historical data query; the basic settings are the main interface of data acquisition and communication settings and acquisition points and acquisition time setting, system fault diagnosis and acquisition of temperature exceeds a predetermined value security alarm function; user information interface is mainly set the login information management tools; interface mainly includes query personnel, memos and other auxiliary functions; system description interface mainly for the introduction, the whole system function specification and other related necessary instructions the launch menu items; the implementation of overall system to exit the command.

\section{Temperature Collection and Analysis}

The realization of temperature acquisition monitoring function mainly includes the design of interface in VB6.0 building structure acquisition interface; the corresponding control menu data connection; software program and lower machine communication, real-time data collection and analysis work and temperature display. The following will be introduced in turn.

The interface is designed and set up by the tool related control and menu editor of VB6.0 and set up the associated project to complete the interface. In the process of setting up the interface, be careful to choose the appropriate controls in the form. The design of the interface includes the following items: the collection design, menu design area (the interface design 4 acquisition area), the collection status display area, real-time display of data acquisition channel design collection 
area, acquisition and communication rate, communication port, alarm temperature setting, speed, time interval of data acquisition.

According to the collection data of the system, the corresponding controls are edited, and the temperature acquisition program is built. Several key points of temperature acquisition is the first, to determine the lower machine CAN intelligent temperature monitoring area (node) can correct normal communication, connected with the PC port initialization, configuration CAN communication parameters, communication is received after the temperature data from the CAN, but this time to pay attention, the computer is not generally received the temperature data, and the initial message frames (also called terminal symbol frame start of frame, frame, ID) data and CRC parity information, so the need for the received packets on the corresponding analysis, find out the decomposition temperature information and reduction, we make it become easy to observe and judge the data form. In the system of temperature data acquisition, first system reset can be initialized. At the same time, the CAN intelligent temperature node device CAN identifier adopts the extended frame format. According to ACR and AMR, we filter and filter the frame ID to achieve two sets of [10] to receive all nodes and select the node information needed.

In the temperature monitoring data transmission using 1 bytes of information, 4 bytes of ID, 8 bytes of data frames, CRC check 1 bytes, including the use of Data0 on behalf of the temperature in the 8 byte frame data in the low eight, data1 represents the temperature of the high eight bits, 6 bytes to data7 data2 the rest of the data to the default value, in order to follow the development of the use of,

To understand these, we can reasonably program in the host computer, analyze the collected data and restore the temperature in the monitoring area, so as to make corresponding agricultural activity decisions. To ensure the stability of agricultural production efficiency is improved.

The function in VB6.0: Public Function ProcOnComm (ByVal No As Integer As Integer) for receiving and analyzing the data, the data received by the HR Static HR As String, defined by the length of comparison and frame alignment to determine a data terminal filter, then the corresponding frame split out frame ID and frame data, temperature data, the temperature data analysis algorithm combined, reduction temperature field. The decision is then made to decide whether to alarm or other operations.

After connecting structures and software programming system function interface, formation temperature acquisition and monitoring interface complete, the interface can be remote real-time monitoring of the 4 regional temperature, a large number of personnel for labor liberation, the application of the system for agricultural personnel better for crop monitoring and adjustment of agricultural policy.

In conclusion, after the design is completed the corresponding region temperature monitoring, realize the acquisition of agricultural monitoring area. The design of other main interfaces of CAN temperature monitoring terminal is mainly about data query, storage, printing and output, as well as the more humane and easy operation of the system.

The realization of 3. monitoring network

With the development of science and technology, the era of digitalization has been coming. In agriculture, the advanced technology can be used to plug traditional agriculture into the wings of science and technology, and form an IOT information network, so as to achieve precise and intensive agricultural production and management, so that we can better keep pace with the times and move forward. CAN bus temperature monitoring system of agriculture will be the site of the temperature is transmitted to the remote monitoring terminal through the Internet, the agricultural personnel in the field can also be in the monitoring center through the large screen Internet or better informed in time for the agriculture, agricultural decision-making, dispatching [6].

The system is tested in laboratory and experimental greenhouse, and compared with the field temperature test data, it is found that the system has good performance, which will pave the way for further improvement and intelligent agricultural management system. 


\section{Conclusion}

The system is easy to maintain, low cost, stable performance, high transmission rate and high accuracy. It can improve the quality of agricultural production, improve the economic benefits of agricultural production and protect the ecological environment. The use of this system can achieve the goal of "high quality, high efficiency, low consumption and safety".

A new technological revolution is taking place in the field of modern agricultural applications. The technology of quasi agriculture and digitalized agriculture has attracted much attention in China. Under the vigorous advocacy of the Ministry of agriculture of China, many universities and research institutes in China have done a lot of research on agricultural modernization under the influence of the international agricultural environment. Future agricultural production will be more intelligent, convenient, efficient and environmentally friendly.

\section{Acknowledgment}

Fund Project:

(1) The education of Shandong province "in 13th Five-Year" general self planning project (Grant No. YC2017078);

(2) Social Science Projects of Shandong (Grant No. 17CJY202)

\section{References}

[1] Lai Q, Wang Y. Design of PV Array Failure Module Monitoring System Based on Temperature Measurement[J]. Computer Measurement \& Control, 2017.

[2] Zeng Y, Yu Y, Pan Y, et al. Design of a logistics monitoring system based on Nios II and Beidou/GPS module[C]// International Conference on Information Management. IEEE, 2017.

[3] Tong X, Chen L, Hua Y, et al. Design on Monitoring System for Inland Lake Water Quality Based on Robotic Fish[J]. Computer Measurement \& Control, 2017.

[4] Samuel Shen, Alan Basist, Allan Howard. Structure of a digital agriculture system and agricultural risks due to climate changes[J]. Agriculture and Agricultural Science Procedia,2010, (09):42-51.

[5] Yang R, Wang X, Guo C, et al. Design and Application of Wireless Sensor Network Environmental Monitoring System Based on ZigBee[J]. Chinese Journal of Electron Devices, 2017.

[6] Robert I Davis, Alan Bruns, Reinder J Bril. Controller area network(CAN) schedulability analysis: refuted, revisited and revised[J]. Real-Time System,2007,35(3):239-272. 